\title{
Rayleigh-Taylor Instability of Magnetized Plasma through Darcy Porous Medium
}

\author{
Gamal Abdallah Hoshoudy \\ Department of Applied Mathematics and Computer Science, Faculty of Science, \\ South Valley University, Kena, Egypt \\ Email: g hoshoudy@yahoo.com
}

Received 26 December 2013; revised 25 January 2014; accepted 23 February 2014

Copyright @ 2014 by author and Scientific Research Publishing Inc.

This work is licensed under the Creative Commons Attribution International License (CC BY). http://creativecommons.org/licenses/by/4.0/

(c) (i) Open Access

\begin{abstract}
Effects of horizontal and vertical magnetic field components on the Rayleigh-Taylor instability of stratified incompressible plasmas layer of variable density through Darcy porous medium are studied. The basic magnetohydrodynamic (MHD) set of equations has been constructed and linearized. Then the linear normalized growth rate is obtained analytically as a function of the physical parameters of the system considered. Numerical calculations have been performed to see the effects of various parameters on the normalized growth rate of Rayleigh-Taylor instability. It is found that the parameter $\lambda^{*}=\lambda L_{D}$ ( $\lambda$ is constant and $L_{D}$ is the density-scale length) has an important role on the happening stability, where the maximum instability comes at $\lambda^{*}=-0.5$ and to get more stable model we select $\lambda^{*}$ through the region $-0.5<\lambda^{*}$ or $\lambda^{*}<-0.5$. The system will be more stable for the positive values of $\lambda^{*}$.
\end{abstract}

Keywords

Rayleigh-Taylor Instability; Magnetized Plasmas; Darcy Porous Media

\section{Introduction}

The hydromagnetics stability of a magnetized plasma of varying density is of considerable importance in several astrophysical situations such as supernova explosions, in heating in solar corona, theories of sunspot magnetic fields, the formation and mixing of clouds and the stability of the stellar atmospheres in magnetic fields.

The classical study of the equilibrium of an incompressible, inviscid fluid of variable density was first undertaken by Rayleigh [1], and later applied to all accelerated fluids by Taylor [2]. Rayleigh showed the equilibrium of a horizontal layer of incompressible, ideal fluid is stable or unstable according as the density increases or de- 
creases anywhere in the vertically upward direction. Since then the problem is called Rayleigh-Taylor instability (RTI). RTI occurs naturally in many phenomenons of astrophysics, geophysics, and laboratories. It derives its character from the adverse density distribution of the matter, where the investigation of Rayleigh-Taylor instabilities in a magnetized plasma is a problem of considerable interest in space (ionospheric spread-F), fusion (curvature induced instabilities like interchange, ballooning, etc.) and the astrophysical plasmas.

Under various physical effects, the Rayleigh-Taylor instability problem of a finite layer of a fluid has been studied by several authors in hydrodynamics and in magnetohydrodynamics domain; the stabilizing effect of magnetic field on RTI problem for an incompressible plasma has been demonstrated by Kruskal and Schwarzschild [3] for a horizontal orientation of the magnetic field and by Hide [4] for a vertical orientation. The effects of viscosity and compressibility on the RTI of stratified plasma in the presence of magnetic field have been studied by Bhatia [5]. The RTI in a rotating plasma of variable density including simultaneously the effects of viscosity and the finiteness of the ion Larmor radius have been investigated by Bhatia and Steiner [6]. The effect of Hall current and finite electrical resistivity on the RTI of viscous, incompressible, finitely conducting plasma in a downward gravitational field under the influence of a uniform magnetic field normal to gravity has been studied by Kamla and Srivastav [7]. The RTI of an infinitely conducting stratified dusty plasma medium including the effects of FLR corrections in the presence of a horizontal magnetic field has been studied by Kamal and Chhajlani [8]. The RTI of a plasma layer in the presence of a horizontal magnetic field is investigated, taking into account the effects of Hall-currents and an arbitrarily large density gradient by Donald [9]. The effects of Hall currents and viscosity on the RTI of an incompressible infinitely conducting stratified plasma permeated by a two-dimensional horizontal magnetic field have been investigated by Ahsan and Bhatia [10]. The effects of Hall currents on the RTI of a finitely conducting stratified partially ionized plasma, where the plasma is permeated by a two dimensional horizontal magnetic field have been studied by Aiyub and Bhatia [11]. In the presence of magnitude of the gravitational acceleration, the RTI of stratified incompressible plasma has been studied by Goldston and Rutherford [12]. The RTI in the presence of horizontal magnetic field of incompressible plasma has been studied by Wu et al. [13].

The RTI of magnetized plasma through porous medium problem has a great scientific interest, where this problem corresponds physically (in astrophysics) to the Rayleigh-Taylor instability of an equatorial section of a planetary magnetosphere or of a stellar atmosphere where the magnetic field is perpendicular or parallels to gravity. So, the RTI of a stratified plasma through porous medium in the presence or absence of magnetic field has been studied by a number of researchers (Chhajlani and Vaghela, Vyas and Chhajlani, Sharma and Bhardwaj, Sharma and Sharma, Sharma and Trilok, Sharma and Sunil, Shikha and Bhatia, Opara, Sharma and Sunil, Sunil and Sharma, Sharma and Thakur and Sharma and Rajput). In this case (Darcy's model), the usual viscous term in the equation of motion is replaced by the resistive term $\left(-\frac{\mu}{k_{1}} \boldsymbol{U}_{a}\right)$, where $\mu$ is the fluid viscosity, $k_{1}$ is the medium permeability and $\boldsymbol{U}_{a}$ is the Darcian (filter) velocity of the fluid.

In all the above-mentioned studies, the behaviour of growth rates is considered with respect to the porosity of porous medium and the medium permeability in the presence of an variable magnetic field in $x$-direction only or in $z$ - direction only. Here, we will discuss the role of resistive term (Darcy's term) besides the components of magnetic field in both $x$-and $z$-direction on growth rates of RTI of plasma layer

\section{Formulation of the Problem}

We consider the strata of incompressible and inviscous plasma as a fluid of electrons and immobile ions through Darcy porous medium in the presence of magnetic field $\boldsymbol{B}$, where the relevant equations may be written, respectively (see references [12]-[25]),

$$
\begin{aligned}
\frac{\rho}{\varepsilon}\left(\frac{\partial}{\partial t}+\frac{\boldsymbol{U}}{\varepsilon} \cdot \nabla\right) \boldsymbol{U} & =-\nabla P+\rho \boldsymbol{g}+\frac{1}{\mu_{e}}(\nabla \times \boldsymbol{B}) \times \boldsymbol{B}-\frac{\mu}{k_{1}} \boldsymbol{U}, \\
\nabla \cdot \boldsymbol{U} & =0 \\
\frac{\partial \boldsymbol{B}}{\partial t} & =\nabla \times\left(\frac{\boldsymbol{U}}{\varepsilon} \times \boldsymbol{B}\right) .
\end{aligned}
$$


For incompressible flow the fluid elements move without changing density is to say that the Lagrangian total derivative of density is zero, that is (see reference [1])

$$
\frac{\mathrm{d} \rho}{\mathrm{d} t}=\left(\frac{\partial}{\partial t}+\frac{\boldsymbol{U}}{\varepsilon} \cdot \nabla\right) \rho=0
$$

where $\boldsymbol{U}$ is the velocity of the fluid, $\rho$ is the density, $p$ thermal pressure, $\mu_{e}$ magnetic permeability, $\mu_{0}$ coefficient of dynamic viscosity and $\boldsymbol{g}$ is the gravitational acceleration.

One can see that the set of Equations (1)-(4) is complete for describing the magnetic field effects on the R-T instability of incompressible plasma, since its number of equations exactly equals its number of unknown quantities: Two unknown vector quantities $\boldsymbol{U}$ and $\boldsymbol{B}$ and two unknown scalar quantities $P$ and $\rho$. For the equilibrium profiles can be expressed in the form $U_{0}=0, \rho_{0}=\rho_{0}(z), p=p_{0}(z)$ and $\boldsymbol{B}_{0}=B_{x 0}(z) \boldsymbol{e}_{x}+B_{z 0}(z) \boldsymbol{e}_{z}$.

Now, we assume a small perturbation in the system of Equations (1)-(4), where the perturbations in the velocity $\boldsymbol{U}$, pressure $p$, magnetic field $\boldsymbol{B}$, and density $\rho$, respectively, are $U=U_{1}(x, y, z, t)$,

$p=p_{0}(z)+p_{1}(x, y, z, t), B=B_{0}(z)+B_{1}(x, y, z, t)$, and $\rho=\rho_{0}(z)+\rho_{1}(x, y, z, t)$. Then, the linearized equations can be easily derived from Equations (1)-(4) in the form

$$
\begin{gathered}
\frac{\rho_{0}}{\varepsilon} \frac{\partial \boldsymbol{U}_{1}}{\partial t}=-\nabla P_{1}+\rho_{1} \boldsymbol{g}+\frac{1}{\mu_{e}}\left[\left(\nabla \times B_{0}\right) \times B_{1}+\left(\nabla \times B_{1}\right) \times B_{0}\right]-\frac{\mu_{0}}{k_{1}} \boldsymbol{U}_{1} \\
\nabla \cdot \boldsymbol{U}_{1}=0 \\
\frac{\partial \boldsymbol{B}_{1}}{\partial t}=\nabla \times\left(\frac{\boldsymbol{U}_{1}}{\varepsilon} \times \boldsymbol{B}_{0}\right) \\
\frac{\partial \rho_{1}}{\partial t}+\left(\frac{\boldsymbol{U}_{1}}{\varepsilon} \cdot \nabla\right) \rho_{0}=0
\end{gathered}
$$

Now, let $\boldsymbol{U}_{1}=\left(u_{x 1}, u_{y 1}, u_{z 1}\right), \quad \boldsymbol{B}_{1}=\left(B_{x 1}, B_{y 1}, B_{z 1}\right), \boldsymbol{g}=(0,0,-g)$ and the fluid is arranged in horizontal strata, then $\rho_{0}$ is a function of the vertical coordinate $z$ only (i.e. $\rho_{0}=\rho_{0}(z)$ ) and $\boldsymbol{B}_{0}=B_{x 0}(z) \boldsymbol{e}_{x}+B_{z 0}(z) \boldsymbol{e}_{z}$. Then the system of Equations (4)-(8) become

$$
\begin{gathered}
\frac{\rho_{0}}{\varepsilon}\left\{\frac{\partial}{\partial t}+\frac{\varepsilon v_{0}}{k_{1}}\right\} u_{x 1}=-\frac{\partial p}{\partial x}+\frac{1}{\mu_{e}}\left\{B_{z 1} \frac{\partial B_{x 0}(z)}{\partial z}+B_{0 z}(z)\left(\frac{\partial B_{x 1}}{\partial z}-\frac{\partial B_{z 1}}{\partial x}\right)\right\} \\
\frac{\rho_{0}}{\varepsilon}\left\{\frac{\partial}{\partial t}+\frac{\varepsilon v_{0}}{k_{1}}\right\} u_{y 1}=-\frac{\partial p}{\partial y}+\frac{1}{\mu_{e}}\left\{B_{x 0}(z)\left(\frac{\partial B_{y 1}}{\partial x}-\frac{\partial B_{x 1}}{\partial y}\right)+B_{0 z}(z)\left(\frac{\partial B_{y 1}}{\partial z}-\frac{\partial B_{x 1}}{\partial y}\right)\right\} \\
\frac{\rho_{0}}{\varepsilon}\left\{\frac{\partial}{\partial t}+\frac{\varepsilon v_{0}}{k_{1}}\right\} u_{z 1}=-\frac{\partial p}{\partial z}+\frac{1}{\mu_{e}}\left\{-B_{x 1} \frac{\partial B_{x 0}(z)}{\partial z}+B_{x 0}(z)\left(\frac{\partial B_{z 1}}{\partial x}-\frac{\partial B_{x 1}}{\partial z}\right)\right\}-\rho_{1} g \\
\frac{\partial B_{1}}{\partial t}=\frac{\partial}{\partial t}\left(B_{x 1}, B_{y 1}, B_{z 1}\right)=\frac{1}{\varepsilon}\left\{\left[-B_{x 1}(z) \frac{\partial u_{y 1}}{\partial y}+\frac{\partial u_{z 1}}{\partial z}=0\right.\right. \\
\left.\left[B_{x 0}(z) \frac{\partial u_{y 1}}{\partial x}-\frac{\partial}{\partial z}\left(B_{z 0}(z) u_{y 1}\right)\right],\left[\frac{\partial}{\partial x}\left(B_{x 0}(z) u_{z 1}-B_{z 0}(z) u_{x 1}\right)-B_{z 0}(z) \frac{\partial u_{y 1}}{\partial y}\right]\right\} \\
\frac{\partial \rho_{1}}{\partial t}+\frac{1}{\varepsilon} \frac{\mathrm{d} \rho_{0}(z)}{\mathrm{d} z} u_{z 1}=0
\end{gathered}
$$

If we assume that the perturbation in any physical quantity takes the form

$$
\psi_{1}(x, y, z, t)=\psi_{1}(z) \exp \left\{\mathrm{i}\left(k_{x} x+k_{y} y-\omega t\right)\right\},
$$


where $k_{x}$ and $k_{y}$ are horizontal components of the wave-number vector $\boldsymbol{k}$ such that $k^{2}=k_{x}^{2}+k_{y}^{2}$ and $\omega$ (may be complex $\left.\left(\omega=\omega_{r}+\mathrm{i} \gamma\right)\right)$ is the frequency of perturbations or the rate at which the system departs from equilibrium thee initial state. Using the expression (15) in the system of Equations (9)-(14), we have

$$
\begin{gathered}
\frac{\rho_{0}}{\varepsilon}\left\{\frac{\varepsilon v_{0}}{k_{1}}-\mathrm{i} \omega\right\} u_{x 1}=-\mathrm{i} k_{x} p_{1}+\frac{1}{\mu_{e}}\left\{B_{z 1} \frac{\partial B_{x 0}(z)}{\partial z}+B_{z 0}(z)\left[\frac{\partial B_{x 1}(z)}{\partial z}-\mathrm{i} k_{x} B_{z 1}\right]\right\}, \\
\frac{\rho_{0}}{\varepsilon}\left\{\frac{\varepsilon v_{0}}{k_{1}}-\mathrm{i} \omega\right\} u_{y 1}=-\mathrm{i} k_{y} p_{1}+\frac{1}{\mu_{e}}\left\{\mathrm{i} B_{x 0}(z)\left[k_{x} B_{y 1}-k_{y} B_{x 1}\right]+B_{z 0}(z)\left[\frac{\partial B_{y 1}(z)}{\partial z}-\mathrm{i} k_{y} B_{z 1}\right]\right\}, \\
\frac{\rho_{0}}{\varepsilon}\left\{\frac{\varepsilon v_{0}}{k_{1}}-\mathrm{i} \omega\right\} u_{z 1}=-\frac{\partial p_{1}}{\partial z}-\rho_{1} g+\frac{1}{\mu_{e}}\left\{-B_{x 1} \frac{\partial B_{x 0}(z)}{\partial z}+B_{x 0}(z)\left[\mathrm{i} k_{x} B_{z 1}-\frac{\partial B_{x 1}(z)}{\partial z}\right]\right\}, \\
-\mathrm{i} \omega\left\{B_{x 1}, B_{y 1}, B_{z 1}\right\}=\frac{1}{\varepsilon}\left\{\left[-\mathrm{i} k_{y} B_{x 0}+\mathrm{i} k_{y} u_{y 1}+\frac{\partial u_{z 1}}{\partial z}=0,\right.\right. \\
\left.\quad\left[\mathrm{i} k_{x} B_{x 0}(z) u_{y 1}-\frac{\partial}{\partial z}\left(B_{z 0}(z) u_{y 1}\right)\right],\left[\mathrm{i} k_{x}\left(B_{x 0}(z) u_{z 1}-B_{z 0}(z) u_{x 1}\right)-\mathrm{i} k_{y} B_{z 0}(z) u_{y 1}\right]\right\}, \\
-i \mathrm{i} \omega \rho_{1}+\frac{1}{\varepsilon} \frac{\mathrm{d} \rho_{0}}{\mathrm{~d} z} u_{z 1}=0,
\end{gathered}
$$

Now, if we eliminate some of the variables from the system of Equations (16)-(21), we have a differential equation in $u_{z 1}$

$$
\begin{gathered}
\left\{\frac{B_{z 0}^{2}(z)}{\mu_{e}}\right\} \frac{\mathrm{d}^{4} u_{z 1}}{\mathrm{~d} z^{4}}+\frac{1}{\mu_{e}}\left\{4 B_{0 z}(z)\left(\frac{\mathrm{d} B_{0}(z)}{\mathrm{d} z}\right)+2 \mathrm{i} k_{x} B_{x 0}(z) B_{z 0}(z)\right\} \frac{\mathrm{d}^{3} u_{z 1}}{\mathrm{~d} z^{3}} \\
+\left\{\left[\frac{\varepsilon v_{0}}{k_{1}}-\mathrm{i} \omega\right] \rho_{0}(\mathrm{i} \omega)+A\right\} \frac{\mathrm{d}^{2} u_{z 1}}{\mathrm{~d} z^{2}}+\left\{\left[\frac{\varepsilon v_{0}}{k_{1}}-\mathrm{i} \omega\right]\left(\frac{\mathrm{d} \rho_{0}}{\mathrm{~d} z}\right)(\mathrm{i} \omega)+B\right\} \frac{\mathrm{d} u_{z 1}}{\mathrm{~d} z}, \\
-\left\{\left[\frac{\varepsilon v_{0}}{k_{1}}-\mathrm{i} \omega\right] \rho_{0}(\mathrm{i} \omega)+g\left(\frac{\mathrm{d} \rho_{0}}{\mathrm{~d} z}\right)-C\right\} u_{z 1}=0
\end{gathered}
$$

\section{A Continuously Stratified Plasma}

In this section we consider the case of incompressible continuously stratified plasma layer of thickness $h$ confined between two rigid boundaries, in which the density and magnetic field distribution are given, respectively, 
by

$$
\rho_{0}(z)=\rho_{0}(0) \exp \left(z / L_{D}\right), B_{x 0}(z)=B_{x 0}(0) \exp \left(z / 2 L_{D}\right) \text { and } B_{z 0}(z)=B_{z 0}(0) \exp \left(z / 2 L_{D}\right)
$$

where $\rho_{0}(0), B_{x 0}(0)$ and $B_{z 0}(0)$ and $L_{D}$ (the density-scale length) are constants, then Equation (22) takes the form

$$
\begin{aligned}
& v_{f_{z}}^{2} \frac{\mathrm{d}^{4} u_{z 1}}{\mathrm{~d} z^{4}}+\frac{2}{L_{D}}\left\{v_{f_{z}}^{2}+\mathrm{i} k_{x} L_{D} v_{f_{z}} v_{f_{x}}\right\} \frac{\mathrm{d}^{3} u_{z 1}}{\mathrm{~d} z^{3}}+\left\{\left[\frac{\varepsilon v_{0}}{k_{1}}-\mathrm{i} \omega\right](\mathrm{i} \omega)-k_{x}^{2} v_{f_{x}}^{2}+\left(\frac{5}{4 L_{D}^{2}}-k^{2}\right) v_{f_{z}}^{2}+\frac{3 \mathrm{i} k_{x} v_{f_{z}} v_{f_{x}}}{L_{D}}\right\} \\
& \cdot \frac{\mathrm{d}^{2} u_{z 1}}{\mathrm{~d} z^{2}}+\frac{1}{L_{D}}\left\{\left[\frac{\varepsilon v_{0}}{k_{1}}-\mathrm{i} \omega\right](\mathrm{i} \omega)-k_{x}^{2} v_{f_{x}}^{2}+\left(\frac{1}{4 L_{D}^{2}}-k^{2}\right) v_{f_{z}}^{2}+\left(\frac{5}{4 L_{D}^{2}}-2 k^{2}\right)\left(\mathrm{i} k_{x} L_{D} v_{f_{z}} v_{f_{x}}\right)\right\} \frac{\mathrm{d} u_{z 1}}{\mathrm{~d} z} \\
& -k^{2}\left\{\left[\frac{\varepsilon v_{0}}{k_{1}}-\mathrm{i} \omega\right](\mathrm{i} \omega)+\frac{g}{L_{D}}-k_{x}^{2} v_{f_{x}}^{2}-\left(\frac{1}{4 k^{2} L_{D}^{3}}-\frac{1}{L_{D}}\right)\left(\mathrm{i} k_{x} L_{D} v_{f_{z}} v_{f_{x}}\right)\right\} u_{z 1}=0 .
\end{aligned}
$$

where $v_{f_{x}}^{2}=\frac{B_{x 0}^{2}(0)}{\mu_{0} \rho_{0}(0)}$ and $v_{f_{z}}^{2}=\frac{B_{z 0}^{2}(0)}{\mu_{0} \rho_{0}(0)}$ are Alfvén velocity.

Now, if we choose $u_{z 1}$ in the form $u_{z 1}=\sin \left(\frac{n \pi}{h} z\right) \exp (\lambda z)$ and by substituting in Equation (24), we will have an equation in both $\sin \left(\frac{n \pi}{h} z\right)$ and $\cos \left(\frac{n \pi}{h} z\right)$. Then coefficients both $\sin \left(\frac{n \pi}{h} z\right)$ and $\cos \left(\frac{n \pi}{h} z\right)$, respectively, are given by:

$$
\begin{aligned}
& v_{f_{z}}^{2}\left\{\lambda^{4}+\left(\frac{n \pi}{h}\right)^{4}-6 \lambda^{2}\left(\frac{n \pi}{h}\right)^{2}\right\}+\frac{2 \lambda}{L_{D}}\left\{v_{f_{z}}^{2}+\mathrm{i} k_{x} L_{D} v_{f_{z}} v_{f_{x}}\right\}\left\{\lambda^{2}-3\left(\frac{n \pi}{h}\right)^{2}\right\} \\
& +\left\{\left[\frac{\varepsilon v_{0}}{k_{1}}-\mathrm{i} \omega\right](\mathrm{i} \omega)-k_{x}^{2} v_{f_{x}}^{2}+\left(\frac{5}{4 L_{D}^{2}}-k^{2}\right) v_{f_{z}}^{2}+\frac{3 i k_{x} v_{f_{z}} v_{f_{x}}}{L_{D}}\right\}\left\{\lambda^{2}-\left(\frac{n \pi}{h}\right)^{2}\right\} \\
& +\frac{\lambda}{L_{D}}\left\{\left[\frac{\varepsilon v_{0}}{k_{1}}-\mathrm{i} \omega\right](\mathrm{i} \omega)-k_{x}^{2} v_{f_{x}}^{2}+\left(\frac{1}{4 L_{D}^{2}}-k^{2}\right) v_{f_{z}}^{2}+\left(\frac{5}{4 L_{D}^{2}}-2 k^{2}\right)\left(\mathrm{i} k_{x} L_{D} v_{f_{z}} v_{f_{x}}\right)\right\} \\
& -k^{2}\left\{\left[\frac{\varepsilon v_{0}}{k_{1}}-\mathrm{i} \omega\right](\mathrm{i} \omega)+\frac{g}{L_{D}}-k_{x}^{2} v_{f_{x}}^{2}-\left(\frac{1}{4 k^{2} L_{D}^{3}}-\frac{1}{L_{D}}\right)\left(\mathrm{i} k_{x} L_{D} v_{f_{z}} v_{f_{x}}\right)\right\}=0, \\
& \quad+2 \lambda\left\{\left[\frac{\varepsilon v_{0}}{k_{1}}-\mathrm{i} \omega\right](\mathrm{i} \omega)-k_{x}^{2} v_{f_{x}}^{2}+\left(\frac{5}{4 L_{D}^{2}}-k^{2}\right) v_{f_{z}}^{2}+\frac{3 i k_{x} v_{f_{z}} v_{f_{x}}}{L_{D}}\right\} \\
& \quad+\frac{1}{L_{D}}\left\{\left[\frac{\varepsilon v_{0}}{k_{1}}-\mathrm{i} \omega\right](\mathrm{i} \omega)-k_{x}^{2} v_{f_{x}}^{2}+\left(\frac{1}{4 L_{D}^{2}}-k^{2}\right) v_{f_{z}}^{2}+\left(\frac{5}{4 L_{D}^{2}}-2 k^{2}\right)\left(\mathrm{i} k_{x} L_{D} v_{f_{z}} v_{f_{x}}\right)\right\}=0 .
\end{aligned}
$$

Now, we define the dimensionless quantities

$$
\begin{aligned}
& \omega^{* 2}=\frac{\omega^{2}}{\omega_{p e}^{2}}, \omega_{f_{x}}^{* 2}=\frac{v_{f_{x}}^{2}}{\omega_{p e}^{2} L_{D}^{2}}, \omega_{f_{z}}^{* 2}=\frac{v_{f_{z}}^{2}}{\omega_{p e}^{2} L_{D}^{2}}, \omega_{\text {Darcy }}^{*}=\frac{\left(\frac{\varepsilon v_{0}}{k_{1}}\right)_{D}}{\omega_{p e}}, \lambda^{* 2}=\lambda^{2} L_{D}^{2}, \\
& h^{* 2}=\frac{h^{2}}{L_{D}^{2}}, k^{* 2}=k^{2} L_{D}^{2}, k_{x}^{* 2}=k_{x}^{2} L_{D}^{2}, g^{*}=\frac{g}{\omega_{p e}^{2} L_{D}}, \omega_{p e}=\left(\frac{\rho_{0} e^{2}}{m_{e}^{2}}\right)^{1 / 2} .
\end{aligned}
$$

Then Equations (25) and (26), respectively, take the form 


$$
\begin{aligned}
& \omega_{f_{z}}^{* 2}\left\{\lambda^{* 4}+\left(\frac{n \pi}{h^{*}}\right)^{4}-6 \lambda^{* 2}\left(\frac{n \pi}{h^{*}}\right)^{2}\right\}+2 \lambda^{*}\left\{\omega_{f_{z}}^{* 2}+\mathrm{i} k_{x}^{*} \omega_{f_{x}}^{*} \omega_{f_{z}}^{*}\right\}\left\{\lambda^{* 2}-3\left(\frac{n \pi}{h^{*}}\right)^{2}\right\} \\
& +\left\{\left[\omega_{\text {Darcy }}^{*}-\mathrm{i} \omega^{*}\right]\left(\mathrm{i} \omega^{*}\right)-k_{x}^{2} \omega_{f_{x}}^{* 2}+\left(\frac{5}{4}-k^{* 2}\right) \omega_{f_{z}}^{* 2}+3 \mathrm{i} k_{x}^{*} \omega_{f_{x}}^{*} \omega_{f_{z}}^{*}\right\}\left\{\lambda^{* 2}-\left(\frac{n \pi}{h^{*}}\right)^{2}\right\} \\
& +\lambda^{*}\left\{\left[\omega_{\text {Darcy }}^{*}-\mathrm{i} \omega^{*}\right]\left(\mathrm{i} \omega^{*}\right)-k_{x}^{2} \omega_{f_{x}}^{* 2}+\left(\frac{1}{4}-k^{* 2}\right) \omega_{f_{z}}^{* 2}+\left(\frac{5}{4}-2 k^{* 2}\right)\left(\mathrm{i} k_{x}^{*} \omega_{f_{x}}^{*} \omega_{f_{z}}^{*}\right)\right\} \\
& -k^{2}\left\{\left[\omega_{\text {Darcy }}^{*}-\mathrm{i} \omega^{*}\right]\left(\mathrm{i} \omega^{*}\right)+g^{*}-k_{x}^{2} \omega_{f_{x}}^{* 2}-\left(\frac{1}{4 k^{* 2}}-1\right)\left(\mathrm{i} k_{x}^{*} \omega_{f_{x}}^{*} \omega_{f_{z}}^{*}\right)\right\}=0, \\
& +2 \lambda^{*}\left\{\left[\omega_{f_{z}}^{* 2}\left\{\lambda^{* 2}-\left(\frac{n \pi}{h^{*}}\right)^{2}\right\}+2\left\{\omega_{f_{z}}^{* 2}+\mathrm{i} k_{x}^{*} \omega_{f_{x}}^{*} \omega_{f_{z}}^{*}\right\}\left\{3 \lambda^{* 2}-\left(\frac{n \pi}{h^{*}}\right)^{2}\right\}\right.\right. \\
& \left.+\left\{\left[\omega_{\text {Darcy }}^{*}-\mathrm{i} \omega^{*}\right]\left(\mathrm{i} \omega^{*}\right)-k_{x}^{2} \omega_{f_{x}}^{* 2}+\left(\frac{5}{4}-k^{* 2}\right) \omega_{f_{z}}^{* 2}+3 \mathrm{i} k_{x}^{*} \omega_{f_{x}}^{*} \omega_{f_{z}}^{*}\right\}-k_{x}^{2} \omega_{f_{x}}^{* 2}+\left(\frac{1}{4}-k^{* 2}\right) \omega_{f_{z}}^{* 2}+\left(\frac{5}{4}-2 k^{* 2}\right)\left(\mathrm{i} k_{x}^{*} \omega_{f_{x}}^{*} \omega_{f_{z}}^{*}\right)\right\}=0 .
\end{aligned}
$$

Now, we put $\omega^{*}=\omega_{r}^{*}+\mathrm{i} \gamma$ and for $\omega_{r}^{*}=0 \quad$ (stable oscillations), then Equations (28) and (29) may be given by:

$$
\begin{aligned}
& \omega_{f_{z}}^{* 2}\left\{\lambda^{* 4}+\left(\frac{n \pi}{h^{*}}\right)^{4}-6 \lambda^{* 2}\left(\frac{n \pi}{h^{*}}\right)^{2}\right\}+2 \lambda^{*}\left\{\omega_{f_{z}}^{* 2}+\mathrm{i} k_{x}^{*} \omega_{f_{x}}^{*} \omega_{f_{z}}^{*}\right\}\left\{\lambda^{* 2}-3\left(\frac{n \pi}{h^{*}}\right)^{2}\right\} \\
& +\left\{\left[\omega_{\text {Darcy }}^{*}+\gamma\right](-\gamma)-k_{x}^{2} \omega_{f_{x}}^{* 2}+\left(\frac{5}{4}-k^{* 2}\right) \omega_{f_{z}}^{* 2}+3 \mathrm{i} k_{x}^{*} \omega_{f_{x}}^{*} \omega_{f_{z}}^{*}\right\}\left\{\lambda^{* 2}-\left(\frac{n \pi}{h^{*}}\right)^{2}\right\} \\
& +\lambda^{*}\left\{\left[\omega_{\text {Darcy }}^{*}+\gamma\right](-\gamma)-k_{x}^{2} \omega_{f_{x}}^{* 2}+\left(\frac{1}{4}-k^{* 2}\right) \omega_{f_{z}}^{* 2}+\left(\frac{5}{4}-2 k^{* 2}\right)\left(\mathrm{i} k_{x}^{*} \omega_{f_{x}}^{*} \omega_{f_{z}}^{*}\right)\right\} \\
& -k^{2}\left\{\left[\omega_{\text {Darcy }}^{*}+\gamma\right](-\gamma)+g^{*}-k_{x}^{2} \omega_{f_{x}}^{* 2}-\left(\frac{1}{4 k^{* 2}}-1\right)\left(\mathrm{i} k_{x}^{*} \omega_{f_{x}}^{*} \omega_{f_{z}}^{*}\right)\right\}=0, \\
& +4 \lambda^{*} \omega_{f_{z}}^{* 2}\left\{\lambda^{* 2}-\left(\frac{n \pi}{h^{*}}\right)^{2}\right\}+2\left\{\omega_{f_{z}}^{* 2}+\mathrm{i} k_{x}^{*} \omega_{f_{x}}^{*} \omega_{f_{z}}^{*}\right\}\left\{3 \lambda^{* 2}-\left(\frac{n \pi}{h^{*}}\right)^{2}\right\} \\
& +2 \lambda^{*}\left\{\left[\omega_{\text {Darcy }}^{*}+\gamma\right](-\gamma)-k_{x}^{2} \omega_{f_{x}}^{* 2}+\left(\frac{5}{4}-k^{* 2}\right) \omega_{f_{z}}^{* 2}+3 \mathrm{i} k_{x}^{*} \omega_{f_{x}}^{*} \omega_{f_{z}}^{*}\right\} \\
& +\left\{\left[\omega_{\text {Darcy }}^{*}+\gamma\right](-\gamma)-k_{x}^{2} \omega_{f_{x}}^{* 2}+\left(\frac{1}{4}-k^{* 2}\right) \omega_{f_{z}}^{* 2}+\left(\frac{5}{4}-2 k^{* 2}\right)\left(\mathrm{i} k_{x}^{*} \omega_{f_{x}}^{*} \omega_{f_{z}}^{*}\right)\right\}=0 .
\end{aligned}
$$

Now, if we rearrange the above two equations (Equations (30) and (31)), we will have

$$
\begin{aligned}
& \left\{\left(\frac{n \pi}{h^{*}}\right)^{2}+k^{* 2}-\lambda^{* 2}-\lambda^{*}\right\} \gamma^{2}+\left\{\left[\left(\frac{n \pi}{h^{*}}\right)^{2}+k^{* 2}-\lambda^{* 2}-\lambda^{*}\right] \omega_{\text {Darcy }}^{*}\right\} \gamma+\left\{\left(\frac{n \pi}{h^{*}}\right)^{2}+k^{* 2}-\lambda^{* 2}-\lambda^{*}\right\} k_{x}^{2} \omega_{f_{x}}^{* 2} \\
& +\left\{\lambda^{* 4}+\left(\frac{n \pi}{h^{*}}\right)^{4}-6 \lambda^{* 2}\left(\frac{n \pi}{h^{*}}\right)^{2}+2 \lambda^{*}\left[\lambda^{* 2}-3\left(\frac{n \pi}{h^{*}}\right)^{2}\right]+\left[\frac{5}{4}-k^{* 2}\right]\left[\lambda^{* 2}-\left(\frac{n \pi}{h^{*}}\right)^{2}\right]+\lambda^{*}\left[\frac{1}{4}-k^{* 2}\right]\right\} \omega_{f_{z}}^{* 2} \\
& +\left\{2 \lambda^{*}\left[\lambda^{* 2}-3\left(\frac{n \pi}{h^{*}}\right)^{2}\right]+3\left[\lambda^{* 2}-\left(\frac{n \pi}{h^{*}}\right)^{2}\right]+\lambda^{*}\left[\frac{5}{4}-2 k^{* 2}\right]+\left[\frac{1}{4}-k^{* 2}\right]\right\}\left\{\mathrm{i} k_{x}^{*} \omega_{f_{x}}^{*} \omega_{f_{z}}^{*}\right\}-k^{* 2} g^{*}=0,
\end{aligned}
$$




$$
\begin{aligned}
& \left\{2 \lambda^{*}+1\right\} \gamma^{2}+\left\{\left[2 \lambda^{*}+1\right] \omega_{\text {Darcy }}^{*}\right\} \gamma-\left\{2 \lambda^{*}+1\right\}\left\{2 \lambda^{* 2}+2 \lambda^{*}+\frac{1}{4}-2\left(\frac{n \pi}{h^{*}}\right)^{2}-k^{* 2}\right\} \omega_{f_{z}}^{* 2} \\
& +\left\{2 \lambda^{*}+1\right\} k_{x}^{* 2} \omega_{f_{x}}^{* 2}-\left\{6 \lambda^{* 2}+6 \lambda^{*}-2\left(\frac{n \pi}{h^{*}}\right)^{2}+\frac{5}{4}-2 k^{* 2}\right\}\left\{\mathrm{i} k_{x}^{*} \omega_{f_{x}}^{*} \omega_{f_{z}}^{*}\right\}=0 .
\end{aligned}
$$

From Equations (32) and (33) maybe we can specialize the next special cases:

(i) In the case of $\omega_{f_{x}}^{* 2}=0, \quad \omega_{f_{z}}^{* 2}=0$ and $\omega_{\text {Darcy }}^{*}=0$

From Equation (33) we get $\lambda^{*}=-\frac{1}{2}$, and substituting in Equation (32) we find that the normalized growth rate given by

$$
\gamma_{\text {Goldston and Rutherford }}=\sqrt{\frac{k^{* 2} g^{*}}{\frac{1}{4}+\left(\frac{n \pi}{h^{*}}\right)^{2}+k^{* 2}}}
$$

This case is considered by Goldston and Rutherford (see reference [12]).

(ii) In the case of $\omega_{f_{x}}^{* 2} \neq 0, \quad \omega_{f_{z}}^{* 2}=0$ and $\omega_{\text {Darcy }}^{*}=0$ A second time, from Equation (33) we get $\lambda^{*}=-\frac{1}{2}$, and substituting in Equation (32), then the normalized
growth rate given by

$$
\gamma_{\text {horizont magnetic field }}=\sqrt{\frac{k^{* 2} g^{*}}{\frac{1}{4}+\left(\frac{n \pi}{h^{*}}\right)^{2}+k^{* 2}}-k_{x}^{* 2} \omega_{f_{x}}^{* 2}} .
$$

This case studied in reference [12]. It is clarified that, the horizontal magnetic field has stabilizing effect on RTI problem. This influence is obvious from Equations (34) and (35), where

$\gamma_{\text {horizont magnetic field }}<\gamma_{\text {Goldston and Rutherford }}$.

(iii) In the case of $\omega_{f_{x}}^{* 2}=0, \omega_{f_{z}}^{* 2} \neq 0$ and $\omega_{\text {Darcy }}^{*}=0$.

A third time, from Equation (33) we get $\lambda^{*}=-\frac{1}{2}$, and substituting in Equation (32), the normalized growth rate given by

$$
\gamma_{\text {vertical magnetic field }}=\sqrt{\frac{g^{*} k^{* 2}}{\frac{1}{4}+\left(\frac{n \pi}{h^{*}}\right)^{2}+k^{* 2}}-\frac{\left\{\left(\frac{n \pi}{h^{*}}\right)^{2}+\frac{1}{4}\right\}\left\{\left(\frac{n \pi}{h^{*}}\right)^{2}+k^{* 2}\right\} \omega_{f_{z}}^{* 2}}{\frac{1}{4}+\left(\frac{n \pi}{h^{*}}\right)^{2}+k^{* 2}}} .
$$

Now, comparing between Equations (34) and (36), someone can observe that, the stabilizing role for the vertical magnetic field on the considerable system, where $\gamma_{\text {vertical magnetic field }}<\gamma_{\text {Goldston and Rutherford }}$.

(iv) In the case of $\omega_{f_{x}}^{* 2}=0, \omega_{f_{z}}^{* 2}=0$ and $\omega_{\text {Darcy }}^{*} \neq 0$

A fourth time, from Equation (33) we get $\lambda^{*}=-\frac{1}{2}$, and substituting in Equation (32), the dispersion rate given by

$$
\gamma^{2}+\left\{\omega_{\text {Darcy }}^{*}\right\} \gamma-\frac{k^{* 2} g^{*}}{\left\{\left(\frac{n \pi}{h^{*}}\right)^{2}+k^{* 2}+\frac{1}{4}\right\}}=0 .
$$

Then, the normalized growth rate becomes

$$
\gamma_{\text {Darcy }}=\frac{-\omega_{\text {Darcy }}^{*}}{2} \pm \sqrt{\left(\frac{\omega_{\text {Darcy }}^{*}}{2}\right)^{2}+\frac{k^{* 2} g^{*}}{\left(\frac{n \pi}{h^{*}}\right)^{2}+k^{* 2}+\frac{1}{4}}} .
$$

From Equations (34) and (38) it is very clear that, $\gamma_{\text {Darcy }}<\gamma_{\text {Goldston and Rutherford }}$. 
The stabilizing effects of the horizontal, vertical magnetic and resistive term, unaccompanied, (above cases (i)-(iv)) on the RTI have been numerically presented in Figure 1.

(v) For the general case $\left(\omega_{f_{x}}^{* 2} \neq 0, \omega_{f_{z}}^{* 2} \neq 0\right.$ and $\left.\omega_{\text {Darcy }}^{*} \neq 0\right)$, if we eliminate the term $\mathrm{i} k_{x}^{*} \omega_{f_{x}}^{*} \omega_{f_{z}}^{*}$ between Equations (32) and (33) the normalized dispersion relation takes in the form.

$$
\zeta_{1} \gamma^{2}+\zeta_{2} \gamma+\zeta_{3}=0
$$

In this case the normalized growth rate given as.

$$
\gamma=\frac{1}{2 \zeta_{1}}\left\{-\zeta_{2} \pm \sqrt{\zeta_{2}^{2}-4 \zeta_{1} \zeta_{3}}\right\}
$$

where

$$
\begin{aligned}
\zeta_{1}= & \left\{\left(\frac{n \pi}{h^{*}}\right)^{2}+k^{* 2}-\lambda^{* 2}-\lambda^{*}\right\}\left\{6 \lambda^{* 2}+6 \lambda^{*}-2 k^{* 2}+\frac{5}{4}-2\left(\frac{n \pi}{h^{*}}\right)^{2}\right\} \\
& +\left\{2 \lambda^{*}+1\right\}\left\{2 \lambda^{*}\left[\lambda^{* 2}-3\left(\frac{n \pi}{h^{*}}\right)^{2}\right]+3\left[\lambda^{* 2}-\left(\frac{n \pi}{h^{*}}\right)^{2}\right]+\lambda^{*}\left[\frac{5}{4}-2 k^{* 2}\right]+\left[\frac{1}{4}-k^{* 2}\right]\right\}, \\
\zeta_{2}= & {\left[\left\{6 \lambda^{* 2}+6 \lambda^{*}-2 k^{* 2}+\frac{5}{4}-2\left(\frac{n \pi}{h^{*}}\right)^{2}\right\}\left\{\left(\frac{n \pi}{h^{*}}\right)^{2}+k^{* 2}-\lambda^{* 2}-\lambda^{*}\right\}\right.} \\
+ & \left.\left\{2 \lambda^{*}+1\right\}\left\{2 \lambda^{*}\left[\lambda^{* 2}-3\left(\frac{n \pi}{h^{*}}\right)^{2}\right]+3\left[\lambda^{* 2}-\left(\frac{n \pi}{h^{*}}\right)^{2}\right]+\lambda^{*}\left[\frac{5}{4}-2 k^{* 2}\right]+\left[\frac{1}{4}-k^{* 2}\right]\right\}\right] \omega_{\text {Darcy }}^{*},
\end{aligned}
$$

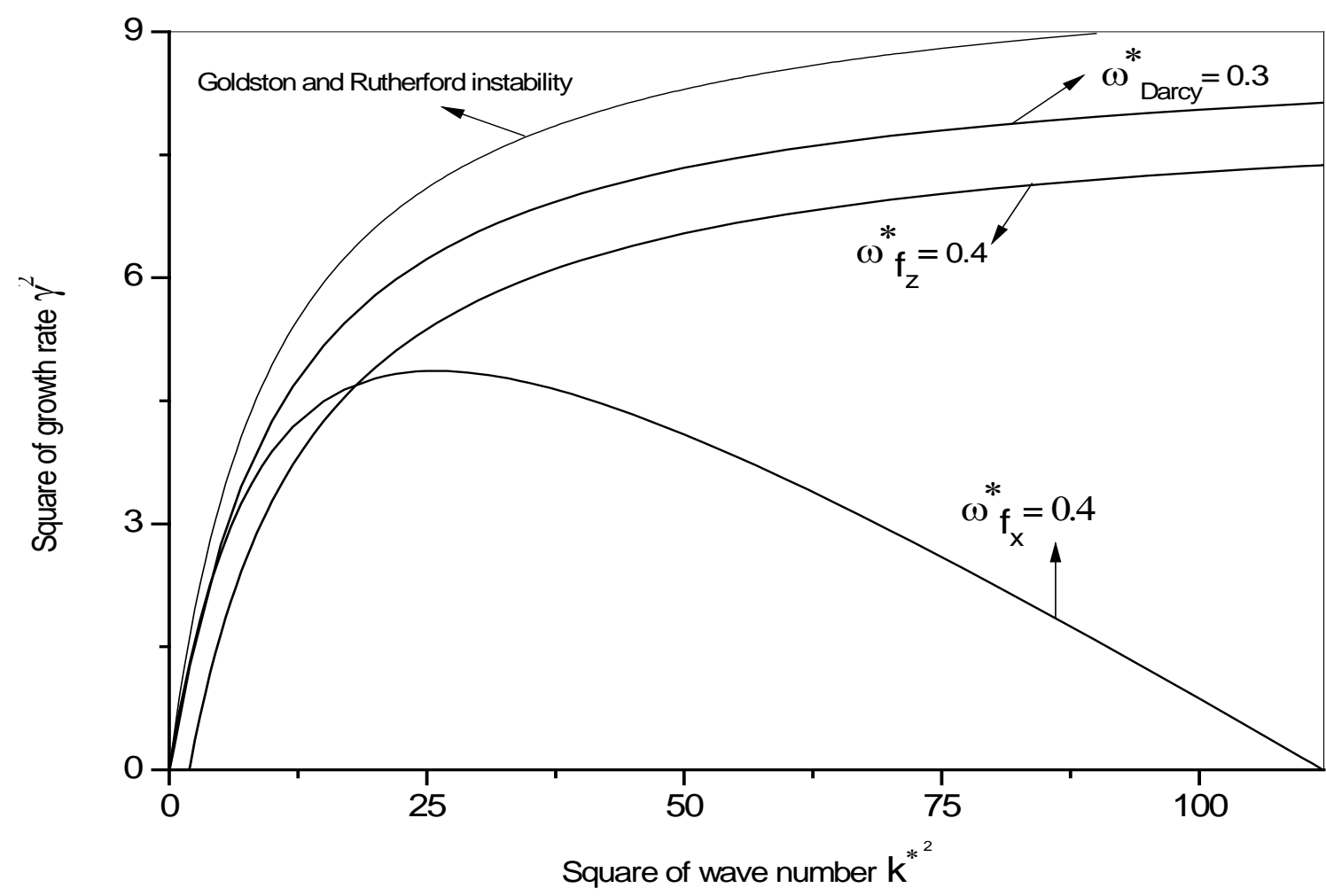

Figure 1. The role of parameter's problem, unaccompanied, where the square normalized growth rate $\left(\gamma^{2}\right)$ against the square normalized wave number $k^{* 2}$ is plotted at $\omega_{f_{x}}^{*}=0.4, \omega_{f_{z}}^{*}=0.4$ and $\omega_{\text {Darc }}^{*}=0.3$. 


$$
\begin{aligned}
\zeta_{3}= & {\left[\left\{6 \lambda^{* 2}+6 \lambda^{*}-2 k^{* 2}+\frac{5}{4}-2\left(\frac{n \pi}{h^{*}}\right)^{2}\right\}\left\{\left(\frac{n \pi}{h^{*}}\right)^{2}+k^{* 2}-\lambda^{* 2}-\lambda^{*}\right\}\right.} \\
& \left.+\left\{2 \lambda^{*}+1\right\}\left\{2 \lambda^{*}\left[\lambda^{* 2}-3\left(\frac{n \pi}{h^{*}}\right)^{2}\right]+3\left[\lambda^{* 2}-\left(\frac{n \pi}{h^{*}}\right)^{2}\right]+\lambda^{*}\left[\frac{5}{4}-2 k^{*^{2}}\right]+\left[\frac{1}{4}-k^{* 2}\right]\right\}\right] k_{x}^{2} \omega_{f_{x}}^{* 2} \\
& \cdot\left[\left\{6 \lambda^{* 2}+6 \lambda^{*}-2 k^{* 2}+\frac{5}{4}-2\left(\frac{n \pi}{h^{*}}\right)^{2}\right\}\right. \\
& \times\left\{\lambda^{* 4}+\left(\frac{n \pi}{h^{*}}\right)^{4}-6 \lambda^{* 2}\left(\frac{n \pi}{h^{*}}\right)^{2}+2 \lambda^{*}\left[\lambda^{* 2}-3\left(\frac{n \pi}{h^{*}}\right)^{2}\right]+\left[\frac{5}{4}-k^{* 2}\right]\left[\lambda^{* 2}-\left(\frac{n \pi}{h^{*}}\right)^{2}\right]+\lambda^{*}\left[\frac{1}{4}-k^{* 2}\right]\right\} \\
& -\left\{2 \lambda^{*}\left[\lambda^{* 2}-3\left(\frac{n \pi}{h^{*}}\right)^{2}\right]+3\left[\lambda^{* 2}-\left(\frac{n \pi}{h^{*}}\right)^{2}\right]+\lambda^{*}\left[\frac{5}{4}-2 k^{* 2}\right]+\left[\frac{1}{4}-k^{* 2}\right]\right\} \\
& \cdot\left\{2 \lambda^{*}+1\right\}\left\{2 \lambda^{*^{2}}+2 \lambda^{*}+\frac{1}{4}-2\left(\frac{n \pi}{h^{*}}\right)^{2}-k^{* 2}\right\}\left[\omega_{f_{z}}^{* 2}-k^{* 2} g^{*}\left\{6 \lambda^{* 2}+6 \lambda^{*}-2 k^{* 2}+\frac{5}{4}-2\left(\frac{n \pi}{h^{*}}\right)^{2}\right\}=0 .\right.
\end{aligned}
$$

In fact, the square of normalized growth rate $\gamma^{2}$ in Equation (40) is a function in the dimensionless quantities of horizontal $\left(\omega_{f_{x}}^{*}\right)$ and vertical $\left(\omega_{f_{z}}^{*}\right)$ components of the magnetic field, the dimensionless resistive term ( $\omega_{\text {Darcy }}^{*}$, dimensionless Darcy term $)$, the wave number $k^{*}$ and $\lambda^{*}\left(\lambda^{*}=\lambda L_{D}\right.$, where $\lambda$ is constant and $L_{D}$ is the density-scale length). The dimensionless quantities $\omega_{f_{x}}^{*}, \omega_{f_{z}}^{*}$ and $\omega_{\text {Darcy }}^{*}$ are the parameters of problem that maybe take a different values. But the constant $\lambda^{*}$ is unknown in the general case (v), while in the absence of magnetic field or in the presence of either them (horizontal or vertical magnetic field components) we note that $\lambda^{*}=-\frac{1}{2}$ (special cases (i)-(iv)). So, firstly we will discuss the role of constant $\lambda^{*}$ on the square of normalized growth rate $\gamma^{2}$ in the presence of horizontal, vertical magnetic field components and resistive term.

The role of constant $\lambda^{*}$ in the presence of both horizontal and vertical magnetic field components $\left(\omega_{f_{x}}^{*}=\omega_{f_{z}}^{*}=0.4\right)$ and resistive term $\left(\omega_{\text {Darcy }}^{*}=0.3\right)$ is plotted in Figure 2, where the square normalized growth

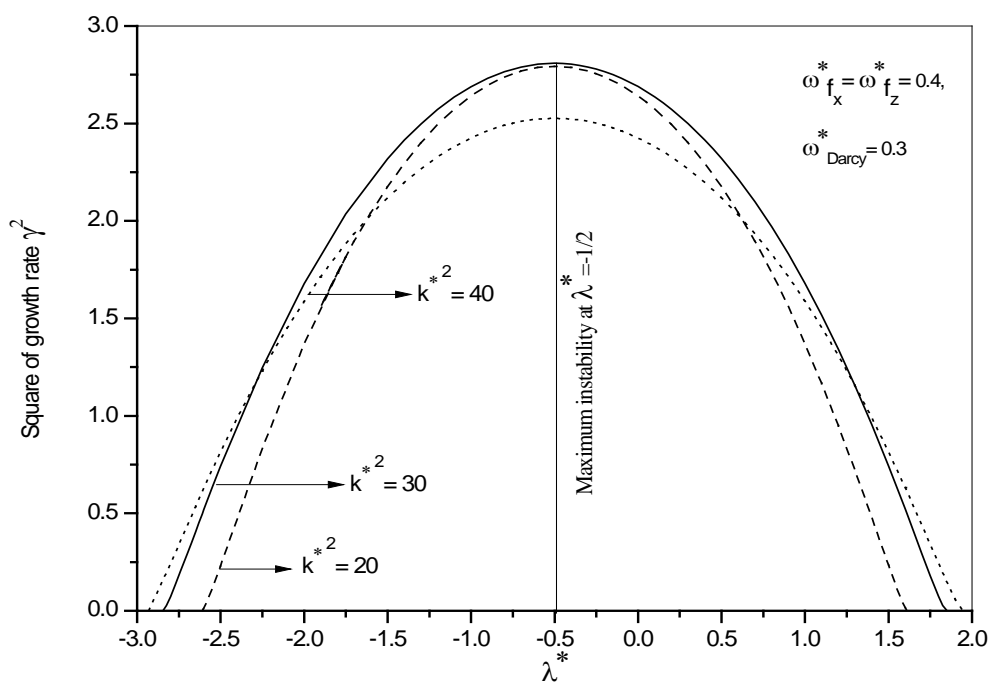

Figure 2. The role of constant $\lambda^{*}$ on the $\gamma^{2}$ in the presence of $\omega_{f_{x}}^{*}=\omega_{f_{z}}^{*}=0.4$ and the resistive term $\omega_{\text {Darcy }}^{*}=0.3$ through the range $\lambda^{*}\left(-3 \leq \lambda^{*} \leq 2\right)$. 
rate $\gamma^{2}$ is plotted against $\lambda^{*}\left(-3 \leq \lambda^{*} \leq 2\right)$ at $k^{* 2}=20,30,40$. For the values $\lambda^{*}$ that is less than $-0.5\left(\lambda^{*}<-0.5\right)$, one can see that, the magnitude of $\gamma^{2}$ decrease with decreasing of $\lambda^{*}$. For the values $\lambda^{*}$ that is greater than $-0.5\left(\lambda^{*}>-0.5\right)$ the magnitude of $\gamma^{2}$ decrease while the magnitude of $\lambda^{*}$ increases. These implies that the maximum instability in the presence of both horizontal and vertical components of magnetic field happens at $\lambda^{*}=-0.5$ (in the presence or absence of resistive term).

The general case (Equation (40)), that gives the effects of horizontal, vertical magnetic field and resistive term together on the instability of the considered system has been presented in Figure 3. where the square normalized growth rate $\gamma^{2}$ is plotted against the square normalized wave number $k^{* 2}$ at $\omega_{f_{x}}^{*}=\omega_{f_{z}}^{*}=0.4, \omega_{\text {Darcy }}^{*}=0.3$ and for different values of $\lambda^{*}(=-1,-0.5,1)$. Second time, one can see that, the maximum instability (maximum square normalized growth rate $\gamma^{2}$ ) happens at $\lambda^{*}=-0.5$ and at $\lambda^{*}=-1$ the magnitudes of $\gamma^{2}$ are less than their counterpart at $\lambda^{*}=-0.5$. Also, the magnitudes of $\gamma^{2}$ at $\lambda^{*}=1$ are less than their counterpart at $\lambda^{*}=-0.5$. Moreovef the magnitudes of $\gamma^{2}$ at $\lambda^{*}=1$ are less than their counterpart at $\lambda^{*}=-1$.

In the case $\lambda^{*}=-\frac{1}{2}$ (maximum instability) the system of Equations (39)-(43) take the form:

$$
\gamma^{2}+\left\{\omega_{\text {Darcy }}^{*}\right\} \gamma+k_{x}^{* 2} \omega_{f_{x}}^{* 2}+\frac{\left[\left(\frac{n \pi}{h^{*}}\right)^{2}+\frac{1}{4}\right]\left[\left(\frac{n \pi}{h^{*}}\right)^{2}+k^{* 2}\right]}{\left(\frac{n \pi}{h^{*}}\right)^{2}+k^{* 2}+\frac{1}{4}} \omega_{f_{z}}^{* 2}-\frac{k^{* 2} g^{*}}{\left(\frac{n \pi}{h^{*}}\right)^{2}+k^{* 2}+\frac{1}{4}}=0 .
$$

Then the maximum normalized growth rate gives by

$$
\gamma_{\max }=-\frac{\omega_{\text {Darcy }}^{*}}{2} \pm \frac{1}{2} \sqrt{\left[\omega_{\text {Darcy }}^{*}\right]^{2}-4\left[k_{x}^{* 2} \omega_{f_{x}}^{* 2}+\frac{\left[\left(\frac{n \pi}{h^{*}}\right)^{2}+\frac{1}{4}\right]\left[\left(\frac{n \pi}{h^{*}}\right)^{2}+k^{* 2}\right]}{\left(\frac{n \pi}{h^{*}}\right)^{2}+k^{* 2}+\frac{1}{4}} \omega_{f_{z}}^{* 2}-\frac{k^{* 2} g^{*}}{\left(\frac{n \pi}{h^{*}}\right)^{2}+k^{* 2}+\frac{1}{4}}\right]} .
$$

Finally, Figure 4 Shows the role of constant $\lambda^{*}$, where the maximum happens at $\lambda^{*}=-0.5$. If we move

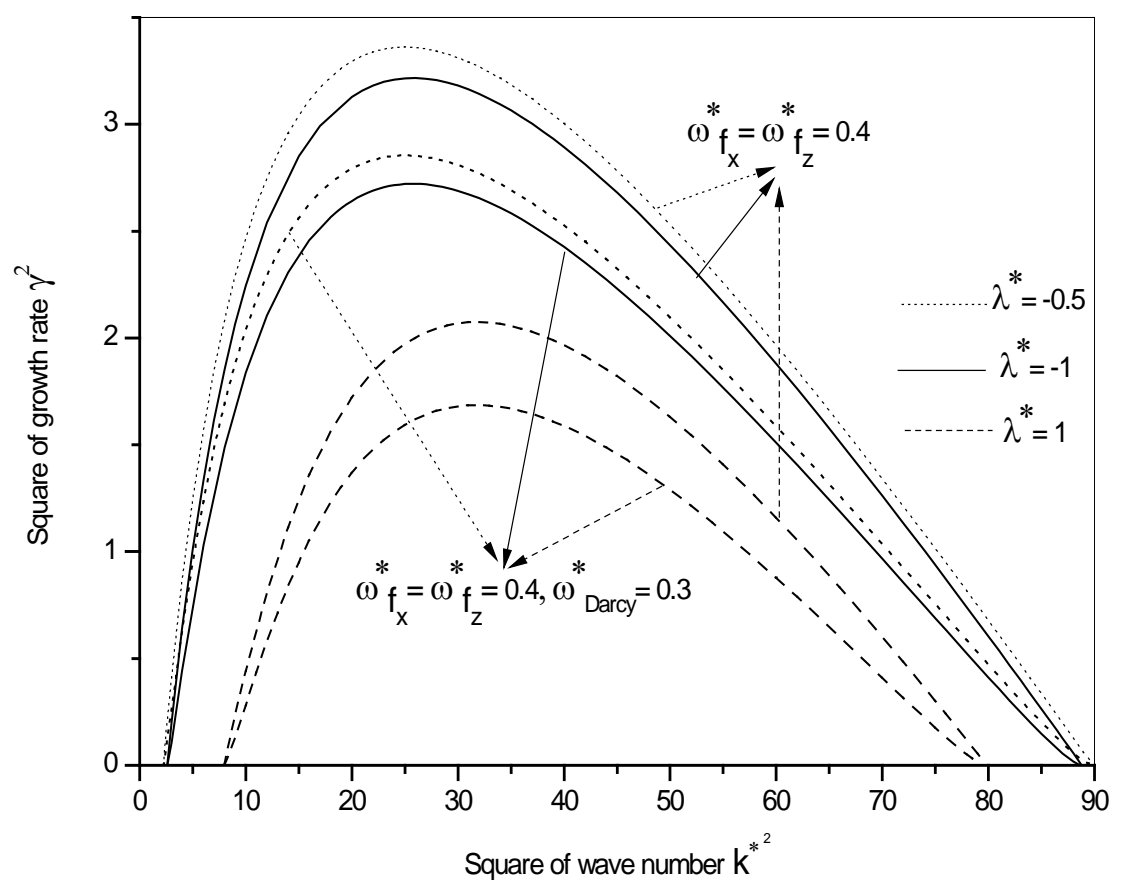

Figure 3. The square normalized growth rate $\left(\gamma^{2}\right)$ against the square normalized wave number $k^{* 2}$ in the presence of $\omega_{f_{x}}^{*}=\omega_{f_{z}}^{*}=0.4$ and the resistive term $\omega_{\text {Darcy }}^{*}=0.3$. 


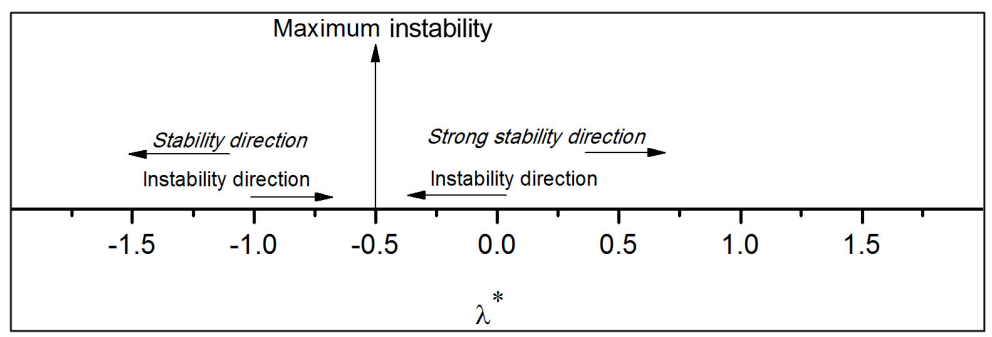

Figure 4. Sketch of the $\lambda^{*}$ through the range $\left(-1.5 \leq \lambda^{*} \leq 1.5\right)$.

toward the point $\lambda^{*}=-0.5$ from the right or left hand the system moves toward instability and vice versa if we move from the point $\lambda^{*}=-0.5$ to the left or right hand, the system tends to stability.

In closing this paper, the Rayleigh-Taylor instability in stratified plasma in the presence of combined effect of horizontal and vertical magnetic field through Darcy porous medium is considered. The solution of the system leads to a dispersion relation where the physical parameters are put in the dimensionless form. Some special cases are particularized to explain the roles that play the variables of the problem and numerical solutions are made. Some stability diagrams are plotted and discussed. The results show that, as the growth rate depends on the horizontal and vertical components of magnetic field and resistive term (Darcy's term) also depends on the parameter $\lambda^{*}=\lambda L_{D}$. Numerically the maximum instability (normalized growth rate) happens at $\lambda^{*}=-0.5$ and then analytically the maximum instability gives in Equation (45). The system will be more stable if we select $\lambda^{*}$ such that to be different than -0.5 . Finally, for the behavior of our selected system with respect to the parameter $\lambda^{*}$ we have not clear interpreting at this stage.

\section{References}

[1] Rayleigh, L. (1882) Proceedings of the London Mathematical Society, 14, 170-177. http://dx.doi.org/10.1112/plms/s1-14.1.170

[2] Taylor, G.I. (1950) Proceeding of the Royal Society of London Series A, 201, 192-196. http://dx.doi.org/10.1098/rspa.1950.0052

[3] Kruskal, M. and Schwarzschild, M. (1954) Proceedings of the Royal Society of London. Series A: Mathematical and Physical Sciences, 223, 348-360. http://dx.doi.org/10.1098/rspa.1954.0120

[4] Hide, R. (1955) Proceedings of the Royal Society of London. Series A: Mathematical and Physical Sciences, 233, 376396. http://dx.doi.org/10.1098/rspa.1955.0273

[5] Bhatia, P.K. (1974) Astrophysics and Space Science, 26, 319-325. http://dx.doi.org/10.1007/BF00645614

[6] Bhatia, P.K. and Steiner, J.M. (1975) Astrophysics and Space Science, 34, 459-465. http://dx.doi.org/10.1007/BF00644812

[7] Abani, K. and Srivastav, K.M. (1975) Il Nuovo Cimento B Series 11, 26, 419-432. http://dx.doi.org/10.1007/BF02738570

[8] Sanghvi, R.K. and Chhajlani, R.K. (1987) Astrophysics and Space Science, 132, 57-64. http://dx.doi.org/10.1007/BF00637781

[9] Donald Ariel, P. (1991) Astrophysics and Space Science, 184, 205-219. http://dx.doi.org/10.1007/BF00642969

[10] Ali, A. and Bhatia, P.K. (1993) Physica Scripta, 47, 567-570. http://dx.doi.org/10.1088/0031-8949/47/4/016

[11] Khan, A. and Bhatia, P.K. (1993) Physica Scripta, 48, 607-611. http://dx.doi.org/10.1088/0031-8949/48/5/017

[12] Goldston, R.J. and Rutherford, P.H. (1995) Introduction to Plasma Physics Institute of Physics, Institute of Physics Publishing, Bristol. http://dx.doi.org/10.1201/9781439822074

[13] Wu, Z., Zhang, W., Li, D. and Yang, W. (2004) Chinese Physics Letters, 21, 2001-2004. http://dx.doi.org/10.1088/0256-307X/21/10/038

[14] Vyas, M.K. and Chhajlani, R.K. (1988) Astrophysics and Space Science, 140, 89-104. http://dx.doi.org/10.1007/BF00643533

[15] Sharma, R.C. and Bhardwaj, V.K. (1990) Czechoslovak Journal of Physics, 40, 753-760. http://dx.doi.org/10.1007/BF01606015

[16] Sharma, R.C. and Sharma, Y.D. (1989) Astrophysics and Space Science, 155, 295-300. 
http://dx.doi.org/10.1007/BF00643865

[17] Sharma, R.C. and Chand, T. (1989) Astrophysics and Space Science, 155, 301-310.

[18] Sharma, R.C. and Sunil (1992) Astrophysics and Space Science, 194, 303-311. http://dx.doi.org/10.1007/BF00643999

[19] Oza, S. and Bhatia, P.K. (1993) Astrophysics and Space Science, 199, 279-288. http://dx.doi.org/10.1007/BF00613201

[20] Opara, F.E. (1994) Astrophysics and Space Science, 213, 197-204. http://dx.doi.org/10.1007/BF00658210

[21] Sharma, R.C. and Sunil (1994) Czechoslovak Journal of Physics, 44, 927-936. http://dx.doi.org/10.1007/BF01715486

[22] Sharma, R.C. and Sunil (1995) Physics of Plasmas, 2, 1886-1892. http://dx.doi.org/10.1063/1.871275

[23] Sunil and Sharma, Y.D. (1996) Polymer-Plastics Technology and Engineering, 35, 221-231. http://dx.doi.org/10.1080/03602559608000580

[24] Sharma, R.C. and Thakur, K.P. (1982) International Journal of Mathematics and Mathematical Sciences, 5, 365-367. http://dx.doi.org/10.1155/S0161171282000350

[25] Sharma, R.C. and Rajput, A. (1992) Astrophysics and Space Science, 187, 105-111. http://dx.doi.org/10.1007/BF00642690 